\title{
A subharmonic detrending or data-smoothing approach for longitudinal road profile measurements
}

\section{Gaunholt, Hans}

Published in:

Proceedings on The 2001 IEEE International Symposium on Circuits and Systems

Link to article, DOI:

10.1109/ISCAS.2001.921095

Publication date:

2001

Document Version

Publisher's PDF, also known as Version of record

Link back to DTU Orbit

Citation (APA):

Gaunholt, H. (2001). A subharmonic detrending or data-smoothing approach for longitudinal road profile measurements. In Proceedings on The 2001 IEEE International Symposium on Circuits and Systems (Vol. 2, pp. 413-416) https://doi.org/10.1109/ISCAS.2001.921095

\section{General rights}

Copyright and moral rights for the publications made accessible in the public portal are retained by the authors and/or other copyright owners and it is a condition of accessing publications that users recognise and abide by the legal requirements associated with these rights.

- Users may download and print one copy of any publication from the public portal for the purpose of private study or research.

- You may not further distribute the material or use it for any profit-making activity or commercial gain

- You may freely distribute the URL identifying the publication in the public portal 


\title{
A SUBHARMONIC DETRENDING OR DATA-SMOOTHING APPROACH FOR LONGITUDINAL ROAD PROFILE MEASUREMENTS
}

\author{
Hans Gaunholt \\ Technical University of Denmark \\ Department of Information Technology, Building 344 \\ DK-2800, Lyngby, Denmark.
}

\begin{abstract}
Commonly used figure-of-merits for longitudinal road profiles such as the International Roughness Index (IRI) and the Ride Number $(\mathrm{RN})$ are based on statistical properties. Raw measured longitudinal road profile data may contain large trending components especially when the measurements are made with walking profilers in hilly regions. To obtain approximate stationary data suitable for statistical processing measurements should be subjected to detrending before calculating the IRI- or $\mathrm{RN}$ - value. In this paper a novel detrending method is proposed which is well suited for removing large profile trends without significantly corrupting the short wavelength content of the profile data. The method called sub-harmonic detrending works by fitting sub-harmonic sinusoids to the data followed by circular filtering in order to remove the trend. The method is also well suited for data-smoothing.
\end{abstract}

\section{INTRODUCTION}

When measuring longitudinal road profiles with certain types of profiling equipment such as walking profilers trends are most often introduced in the measured data. In order to be able to use statistical methods to characterise road profiles we have to obtain data which obviously are more close to be stationary than the data shown in Figure 1. As further described in [1] and [2]

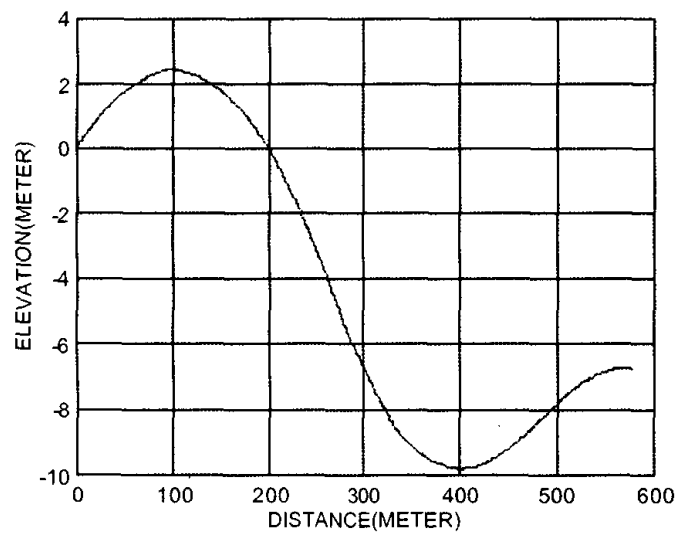

Figure 1: Typical mountain profile the purpose of removing trend is to obtain approximate stationary data prior to calculation of statistical profile indices such as IRI (International Roughness Index) and RN (Ride Number). Otherwise those profile indices may be quite misleading. A typical example of a road profile with trend is shown on Figure 1. The measurements of this profile are made by a walking profiler and are made available for this project by Transit New Zealand. The conventional way of removing trend a process normally called detrending may be performed by doing data antismoothing by linear high pass filtering or data anti-smoothing by spline approximation or polynomial curve fitting as described in [1]. Especially the polynomial curve fitting is very popular but with the drawbacks of smoothing the data with unbounded functions as well as being numerically ill-conditioned. From a road profiling point of view the long wavelength contamination of the shorter wavelengths by the detrending processes may give rise to considerable errors in the calculated roughness index.

In this paper an alternative detrending method is proposed based on sub-harmonic fitting and circular filtering. First the measured data is fitted in a bounded and smooth way by the aid of subharmonic sinusoids. By this we are artificially increasing the observation length creating a continuos and smooth periodic function well suited for DFT-processing. In the actual case the increase is by a factor of four well knowing that the result is dependent on the way this is done. The difference between the true profile prolonged with the artificial profile and the trending sub-harmonic sinusoids is called the sub-harmonic detrended function. The detrended function. however, is the function that appear after circular filtering of sub-harmonic detrended function.

\section{2: SUB-HARMONIC FITTING}

The method works by fitting the profile with sub-harmonic sinusoids $\alpha_{\mathrm{s}}(c)$ of the following form:

$$
\begin{aligned}
\alpha_{s}(c)= & A_{0}+A_{1} \cdot \cos \left(\frac{\pi}{\ell_{0}} \ell+\varphi_{1}\right)+A_{3} \cdot \cos \left(\frac{3 \pi}{2 \ell_{0}} \ell+\varphi_{3}\right) \\
& +A_{2} \cdot \cos \left(\frac{\pi}{2 \ell_{0}} \ell+\varphi_{2}\right)
\end{aligned}
$$


$C_{0}$ is the total length in meters of the measured profile and $C$ is the running length. $A_{0}, A_{1}, A_{2}, \varphi_{1}, \varphi_{2}$ and $\varphi_{3}$ are free parameters which are defined by the approximation process. More subharmonic terms may be added but in the present case it has been found appropriate to consider the above four terms. Notice that the sub-harmonic sinusoidal function $\alpha_{s}(\ell)$ is periodic with a period four times the total length $C_{0}$.

Two solutions for the unknown variables above have been investigated:

1. Two sub-harmonics. By setting $A_{0}$ a free parameter, $A_{3}=0$ and $\varphi_{3}=0$ it is possible to find an analytic solution for the rest of the parameters $A_{1}, A_{2}, \varphi_{1}$ and $\varphi_{2}$ by applying the constraint that the function values and the values of the firstorder derivatives at the endpoints are known.

2. Tree sub-harmonics. All parameters including $A_{3}$ and $\varphi_{3}$ are defined by applying the constraints that the sub-harmonic function $\alpha_{s}(C)$ should fit the measured profile at two intermediate points i.e. for $\epsilon=\epsilon_{0} / 3$ and $\epsilon=2 C_{0} / 3$ as well as satisfying the constraints corresponding to case 1 .

\subsection{TWO SUB-HARMINICS}

By considering the DC-level $\mathrm{A}_{0}$ as a free parameter the following four equations may be set up corresponding to case 1 .

$$
\begin{aligned}
& \alpha(0)-A_{0}=A_{1} \cos \varphi_{1}+A_{2} \cos \varphi_{2} \\
& \alpha\left(\ell_{0}\right)-A_{0}=-A_{1} \cos \varphi_{1}-A_{2} \sin \varphi_{2} \\
& \frac{\ell_{0}}{\pi} \alpha^{\prime}(0)=-A_{1} \sin \varphi_{1}-\frac{1}{2} A_{2} \sin \varphi_{2} \\
& \frac{\ell_{0}}{\pi} \alpha^{\prime}\left(\ell_{0}\right)=A_{1} \sin \varphi_{1}-\frac{1}{2} A_{2} \sin \varphi_{2}
\end{aligned}
$$

The above four equations are readily solved for the unknown parameters $A_{1}, \varphi_{1}, A_{2}$ and $\varphi_{2}$ with the following result.

$$
\begin{aligned}
& \varphi_{2}=-\frac{\pi}{4}+\tan ^{-1}\left(-\frac{2 \ell_{0}}{\pi} \cdot \frac{\alpha^{\prime}(0)+\alpha^{\prime}\left(\complement_{0}\right)}{\alpha(0)+\alpha\left(\ell_{0}\right)-2 \mathrm{~A}_{0}}\right) \\
& A_{2}=\frac{\alpha(0)+\alpha\left(c_{0}\right)-2 A_{0}}{\sqrt{2} \cdot \cos \left(\varphi_{2}+\frac{\pi}{4}\right)} \\
& \varphi_{1}=\tan ^{-1}\left(-\frac{\frac{\ell_{0}}{\pi} \cdot \alpha^{\prime}(0)+\frac{1}{2} A_{2} \sin \varphi_{2}}{\alpha(0)-A_{0}-A_{2} \cos \varphi_{2}}\right) \\
& A_{1}=\frac{\alpha(0)-A_{0}-A_{2} \cos \varphi_{2}}{\cos \varphi_{1}}
\end{aligned}
$$

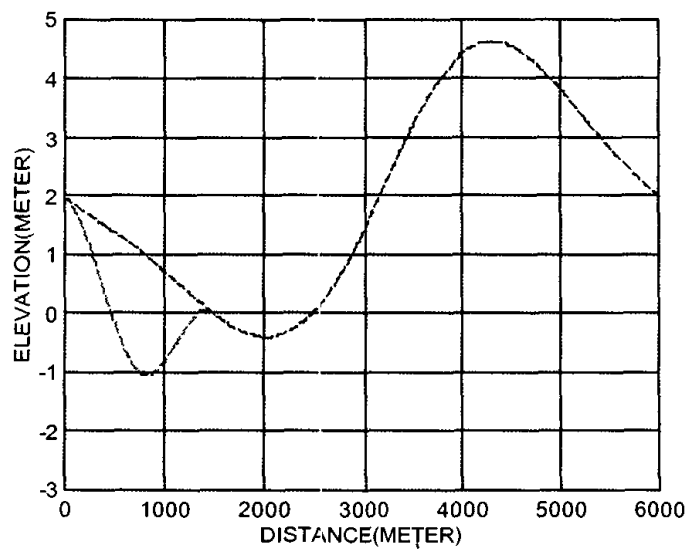

Figure 2. Artificial profile $\alpha_{\mathrm{a}}(\ell)$ and fitted sub-hamonic sinusoids. Two sub-harmonics.

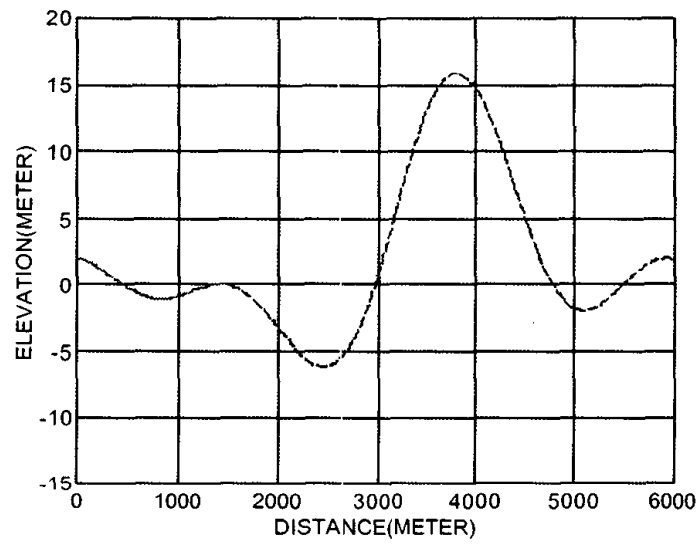

Figure 3. Artificial profile $\alpha_{\mathrm{a}}(c)$ and fitted sub-harmonic sinusoids. Three sub-harmonics.

\subsection{THREE SUB-HARMONICS}

In this case we are obtaining six equations with the six unknowns parameters $A_{1}, \varphi_{1}, A_{2}, \varphi_{2}, A_{3}$ and $\varphi_{3}$ considering the DC-level $A_{0}$ a free parameter. The two additional equations are obtained by adding the constraint that the subharmonic function should fit the data at $C=C_{0} / 3$ and at $C=2 C_{0} / 3$. Due to the limited space available those six equations will not be shown in this paper. It has, however, not been possible to find an analytical solution for the six parameters. Instead a Newton iteration has been applied using as starting values the analytic solution from subsection 2.1. The details as well as the convergence properties of the Newton iteration shall not be considered further in this paper, but the process has been found to work quite satisfactory in solving the above described class of problems. 


\section{ARTIFICIAL PROFILE}

The sub-harmonic fitting processes described above have been tested on an artificial profile,

$$
\alpha_{\mathrm{a}}(\ell)=1-\cos \left(\pi\left(\frac{2 \ell}{\epsilon_{0}}-1\right)\right)-\frac{2 \ell}{\ell_{0}}
$$

with the profile length: $\ell_{0}=1500$ meter. Notice that $\alpha_{\mathrm{a}}(\ell=0)=2$ and $\alpha_{\mathrm{a}}\left(\ell=\ell_{0}\right)=0$. In Figure 2 and Figure 3 are shown the sub-harmonic functions together with the artificial profile corresponding to fitting method case 1 and case 2 . Notice that both functions are periodic with a period of 6000 meter or 4 times the profile length $C_{0}$. The main advantage of the described fitting process has been to create a continuos periodic function with continuos derivatives. By this the Fourier series expansion converges much faster than without fitting with less contamination of the high frequency content. The computation of the Fourier series or spectrum by a conventional DFT [7] requires a four times longer expansion. This obviously is a drawback but with modern computing technology this is not a serious drawback. The advantage of applying subharmonic fitting followed by subharmonic detrending compared with conventional windowing methods $[4,5,6]$ cannot be overestimated. Which of the fitting methods should be applied depend on the appropriate application.

\section{MEASURED PROFILE}

Now let us return to the measured mountain profile from Figure 1. Due to the short wavelength content of the profile we have to estimate the endpoints and the first-order derivatives by some simple smoothing process. We have chosen to fit in a leastsquare way two straight lines to the measured data. One to the first 2.5 meter ( 10 sample points) of the measured profile and one to the last 2.5 meter. The first-order derivatives of the subharmonics are estimated as the slope of these lines. Also the endpoints are replaced by the values determined by the lines. By this a small change of the first and last sample may be expected avoiding abrupt changes in the detrended data. In Figure 4 and 5 are shown sub-harmonic fitting curves corresponding to case 1 . and case 2. described above. It is noticeable that case 2 . with three sub-harmonics gives a better approximation in the profile band but much larger oscillations outside the band. This also holds true when comparing with the artificial profile in Figure 3. As the outside-band trend may be considered an attempt to extrapolate the true profile it appear to be reasonable to chose a method that keeps the outside-band oscillation at a realistic level at the expense of an extended inside-band trending error.

We are compromising by using case 1 . with two sub-harmonics in the following. Now let us compute the (DFT) of the difference between the mountain profile above and the subharmonic fitting functions corresponding to Figure 4 . We are using a DFT that is four times as long as the mountain profile itself with the difference function or sub-harmonic detrended function padded with zeros from the end of the measured profile until the end of the sub-harmonic function. As the length of the extended profile is 2306 meter a removal of the first 23 harmonics from the DFT followed by an inverse DFT correspond to complete removal of all wavelength greater than or equal to 100 meter from the measured profile. Notice that wavelength from 96 meter and shorter are preserved. The result of this process also named circular filtering or circular antismoothing is shown on Figure 6 and Figure 7.

It is noticeable that the introduction of the sub-harmonic trending function and the removal of this function by circular filtering do not contaminate the short wavelength contend of the profile. On the other hand it must be explicitly stated that the actual selection of the trending function indirectly influences the result of the filtering process. If we as an alternative had selected a trending function according to case 2 . with three sub-harmonics a slightly different result of the anti-smoothing process would have been obtained at the long wavelength end of the anti-smoothed

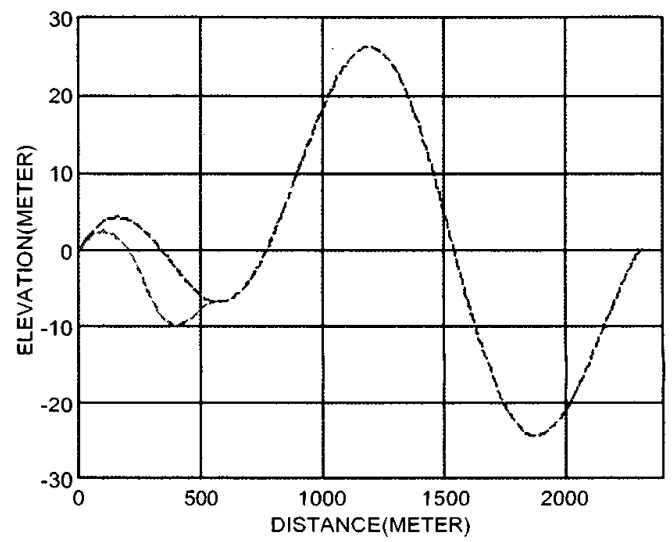

Figure 4. Mountain profile and fitted sub-hamonic sinusoids. Two sub-harmonics.

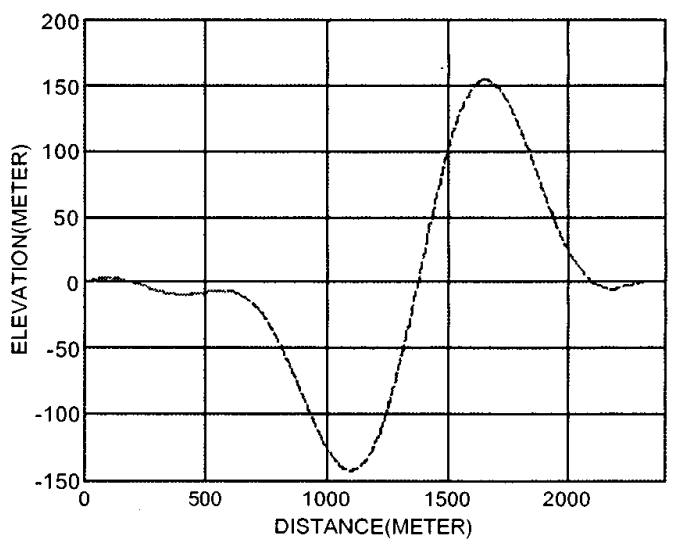

Figure 5. Mountain profile and fitted sub-harmonic sinusoids. Three sub-harmonics. 


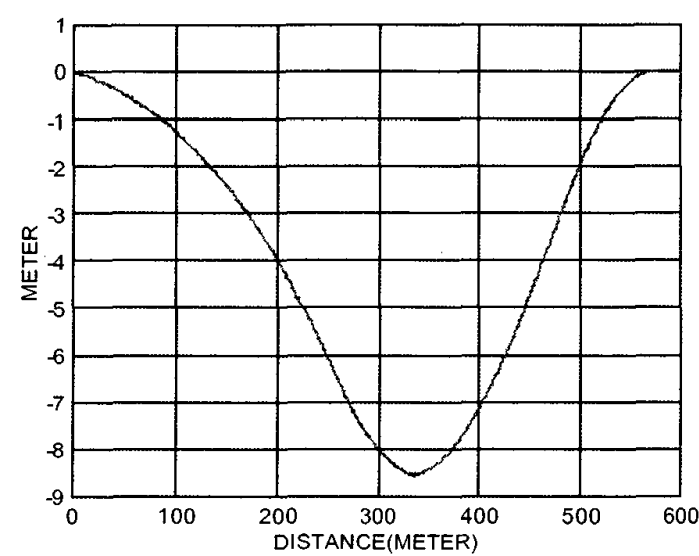

Figure 6. Subharmonic detrended and smoothed detrended profile. Smoothing is performed by deleting all harmonics greather than 23 or wavelengths shorter than 100 meter.

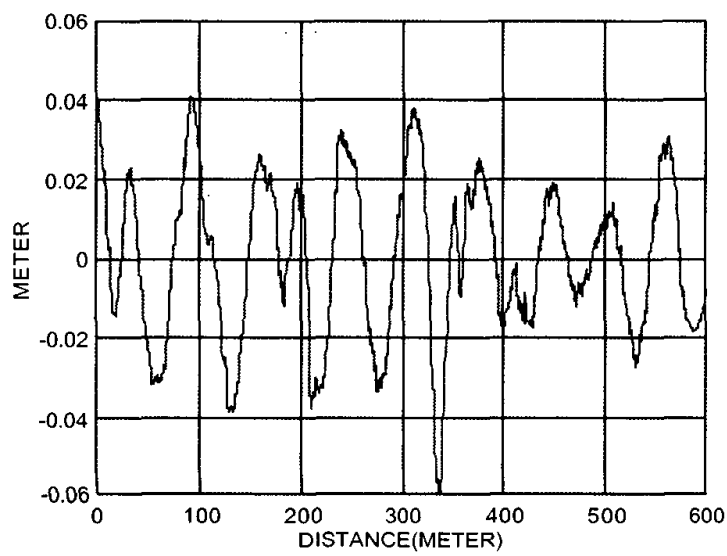

Figure 7. Anti-Smoothed function or difference between the true profile and the smoothed detrended profile from Figure 6.

function.

On Figure 6 it is difficult to see the difference between the subharmonic detrended and smoothed detrended profile, but the difference is shown in Figure 7. The result shown in Figure 7 is our final result concerning anti-smoothing or removal of long wavelengths from the short road profile shown in Figure I. It is also rather surprising that the resulting anti-smoothed or detrended function shown in Figure 7 is quite insensitive to the selection of the sub-harmonic detrending function corresponding to the fitting of two or three sub-harmonic functions as discussed in subsection 2. This only holds true when we are removing the same number of harmonics by circular filtering. The optimal selection of sub-harmonic function as well as the estimation and introduction of higher order sub-harmonic derivatives are still subject to investigation.

\section{CONCLUSION}

In this paper a DFT-based method of smoothing or antismoothing of short road profiles is proposed as an alternative of using conventional linear filkering, polynomial approximation or spline approximation. The method is based on fitting the measured road profile with a sub-harmonic sinusoidal function. A smooth detrended and artificially prolonged profile is obtained with a period four times the observation length. This transformed profile is well suited for circular filtering by the aid of DFT processing. Due to the avoidance of Gibbs phenomenon contamination of the short wavelengths content of the profile is avoided. But the result at longer wavelengths are dependent on the chosen sub-harmonic function. The method has shown excellent results in removing large trends in short road profiles measured in hilly regions. Further improvement of the proposed method may be expected e.g. by fitting more sub-harmonics in order to smooth higher order derivatives.

\section{LITERATURE}

[1] Michael W. Sayers and Steven M. Karamihas, "The Little Book of Profiling" UM'TRI The University of Michigan Transportation Research Institute, September 1998.

[2] Michael W. Sayers, "On the Calculation of IRI from Longitudional Road Profile" Transportation Research Record 1501, Transportation Research Board, National Research Council, Washington D.C., 1995.

[3] Mischa Schwartz, L. Shaw, "Signal Processing: Discrete Spectral Analysis, Detection and Estimation", McGrantHill, Inc, 1975.

[4] Sanjit K. Mitra, "Digital Signal Processing - A ComputerBased Approach". McGrawHill 1998.

[5] John G. Proakis, Dimitris G. Manolakis, "Digital Signal Processing", Prentice-Hall, Inc. 1996.

[6] R.W.Hamming, "Digital Filters" Prentice Hall 1989.

[7] J.W.Cooley and J.W.Tukey:"An algorithm for machine calculation of complex Fourier series" Math. Computation. Vol.19, pp. 297-301, April 1965. 